\title{
Comercio de lanas, estructura agraria y oligarquía en el sur del Perú Nils Jacobsen
}

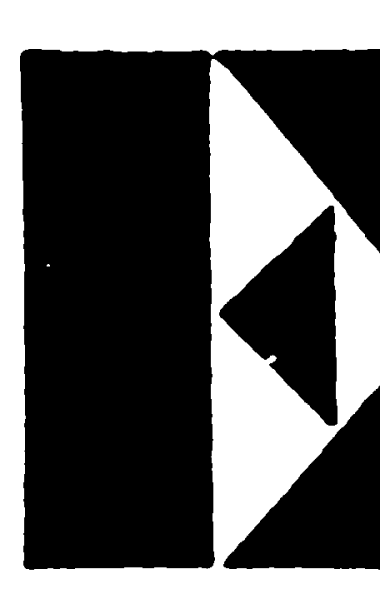
1 desarrollo económico en el sur del Perú hacia fines del siglo XIX y comienzos del siglo XX, un período en que la exportación de la lana fue de importancia determinante, representa un problema especial para la historia peruana: ¿Por qué -en las haciendas ganaderas y las comunidades indígenas - se mantuvieron tan tradicionales y arcaicas las estructuras de la producción agraria, sin que se produjeran inversiones de capitales, se intensificara los métodos de producción o se introdujeran relaciones de trabajo asalariado en las grandes propiedades agrícolas? Este desarrollo es evidentemente muy diferente del de todos los otros sectores de exportación en el Perú hasta 1930, como el azucarero, algodonero, petrolero y minero, donde se realizaron inversiones y en parte se llegó a una influencia decisiva del capital extranjero o al menos -como en la producción algodonera de la zona costera central-a considerables cambios en la organización social y técnica de la producción.

Manuel Burga y Wilson Reategui, historiadores de la Universidad San Marcos, que ya se han distinguido por trabajos referentes a la historia agraria peruana, así como también (Burga junto con Alberto Flores Galindo) por una interesante interpretación de la época de la "República Aristocrática", ahora han intentado encontrar explicaciones para la problemática especial de la economía del sur peruano entre 1890 y 1930 mediante un análisis del comercio de esa época en la región. Su trabajo sobre la casa co: mercial Ricketts en Arequipa entre 1895 y 1935 y sobre sus negocios de exportación de lanas y venta de productos textiles y de ferretería extranjeros y peruanos hacia el interior del país, es mucho más que sólo la historia de una empresa cualquiera. Inspirados metodológicamente por la escuela francesa de los Annales, los autores investigan el ritmo coyuntural del comercio de la lana y otras corrientes mercantiles de la región, las estructuras del mercado en sus diversos niveles entre los productores agrarios -consumidores y los exportadores de lanas-comerciantes mayoristas, la estructu- 
ración espacial de los diferentes ciclos comerciales en el sur del Perú como asimismo, las alianzas y conflictos entre los grupos sociales involucrados en este comercio.

Pero la meta última de este minucioso análisis del comercio sudperuano es una explicación más general de la interacción entre esferas de mercado y de producción, que pretende dar una respuesta a la pregunta planteada inicialmente sobre las causas y razones por las cuales se mantuvieron durante tanto tiempo las relaciones tradicionales de producción extensiva y la pobreza de los campesinos.

En mi opinión, el problema principal del libro parece radicar en las contradicciones que contiene, en el desarrollo de esquemas interpretativos contrapuestos, que en parte van hasta la descripción empírica. Como demostración para lo que afirmo, abordemos el conjunto central de aspectos del que recién hemos hecho mención, la interacción entre las esferas comerciales y de producción.

Por un lado, Burga y Reátegui explican el atraso persistente de las relaciones de producción agrarias y la pobreza de los campesinos en las regiones de producción de lana en el sur del Perú mediante la dependencia absoluta de los precios de la lana fijados en Liverpool y que no se orientaban en los gastos de producción en Puno, Cusco o Arequipa (p. 183). Este sistema de precios, en el cual, según los autores, se fijaban los precios pagados en Arequipa según las fluctuaciones del mercado mundial y en el que los productores, no los consumidores, debían pagar todos los gastos de transporte y de elaboración, las ganancias de los intermediarios $\mathrm{y}$, adicionalmente, los gravámenes tributarios impuestos por el Estado peruano en los años 20, no dejaban a los hacendados y campesinos indígenas utilidades suficientes de sus ventas de lana, como para modernizar considerablemente la estructura de producción mediante inversiones de capital y para estimular el mercado interno (p. 83 y 101). Según este modelo de interpretación, esto significa, que la integración del sur peruano al mercado mundial de la lana no sólo influenció el ritmo de la economía agraria regional, sino también tuvo como consecuencia la perpetuación o -según la terminología de los autores- la reproducción de las estructuras arcaicas de producción y la pobreza campesina.

Pero por otro lado, en determinado contexto, los autores escogen un modelo de explicación totalmente diferente para el mismo fenómeno. Según éste, las causas para la perpetuación de estructuras sociales tradicionales en el sur del Perú estaban en las relaciones de producción mismas: ". . la explotación y la pobreza del campesinado no se realizaba fundamentalmente en el mercado, sino dentro de las haciendas" (p. 108). Sólo una transformación y re- 
volución en las relaciones de producción podrían haber superado la hipodinamia del mercado interno (p. 170). Vale decir, que aquí los autores han invertido la variable dependiente e independiente.

Esto lleva a una serie de aseveraciones totalmente contrarias en el transcurso del libro. Así, los autores por una parte se basan p.e. en la situación en la hacienda Lauramarca, para comprobar que los hacendados en general obligaban a sus colonos a venderles la lana de su propio ganado huaccho a precios muy por debajo del precio de mercado, para revenderlo en seguida a los comerciantes a precios muchísimo más altos (p. 108). Pero cinco páginas antes habían descrito, cómo los "indios de finca" principalmente, es decir los mismos colonos, vendian la lana de sus propios rebaños a los rescatistas, los comerciantes locales de la lana, en los mercados dominicales de la región (p. 103). De hecho parece, que sólo muy pocos terratenientes pudieron obligar a sus colonos, a venderles directamente sus productos ganaderos a precios infimos. Por lo tanto, si se pretende explicar los márgenes estrechos del mercado en el sur peruano mediante la estructura social misma -y esto parece ser razonable-, entonces no es posible aducir el control de los hacendados sobre la comercialización de los productos campesinos, sobre todo, como documentan Burga y Reátegui, dado que solamente una minoría de los campesinos indígenas eran colonos en haciendas ganaderas. Aquí es más bien preciso referirse a las estructuras socio-políticas de poder que ya existían en el sur peruano -y esto es decisivo- al comenzar la fase expansiva de las exportaciones de lana a fines de los años 50 del siglo XIX.

Referente a la fijación de precios de la lana también existen contradicciones. Por un lado, Burga y Reátegui relatan la práctica de la firma Ricketts, de hacer sus compras de lana a través de agentes de negocios en el interior del país, sólo a precios que diariamente se transmitían telegráficamente y que correspondían exactamente a las cotizaciones de Liverpool (p. 83). Pero por otro lado los autores aclaran, que esta importación casi mecánica de los precios de la lana era una característica particular de la estrategia comercial temerosa de la firma Ricketts, cuya meta no era la expansión en el mercado. La competencia entre varios otros mayoristas, en cambio, como p. e. Gibson y Stafford (ambos exportaban más lana que Ricketts), "con frecuencia" llevó a "que los precios no se nivelaran en relación a las cotizaciones del mercado internacional, sino de acuerdo a la competencia interna* (p. 84). Pero incluso esto no podía neutralizar la dependencia intríseca de las fluctuaciones internacionales en los precios. Sin embargo, parece haber existido un nivel secundario de fijación de precios en los centros donde se compraba la lana. 
Estrechamente vinculado a los mecanismos de fijación de precios está el problema del rol de los mayoristas en el desarrollo económico y social general del sur peruano entre los años 1890 y 1930. También aquí, Burga y Reátegui reflejan una imagen que no está libre de contradicciones y que por otra parte, en algunos aspectos fundamentales, no corresponde exactamente a la realidad. Según los autores, el comercio de lanas e incluso, aunque en escala menor, el comercio de productos manufacturados que padecía la eterna morosidad de los pequeños comerciantes, producía rendimientos seguros para los mayoristas. Como esta rentabilidad habría sido constantemente más alta que la de la ganadería, los mayoristas no habrían hecho inversiones en la producción de la lana misma (p. 125). Basándose en el ejemplo de la familia Ricketts, los autores afirman, que los mayoristas se conformaban con invertir sus ganancias en la compra de lujosas villas en Arequipa y en la educación de sus hijos en el extranjero (p. 67).

La idea que el comercio mayorista en Arequipa haya producido rendimientos seguros casi automáticos durante decenios, no parece ser realista. Ya durante la corta crisis económica en 1920/21, algunos exportadores de lana hicieron bancarrota (Appleby, p. 64) y otros, como p.e. Emmel y las Arequipa Agencies se retiraron de la exportación de lanas en los años siguientes. Burga y Reátegui mismos relatan, que la firma J.M. Forga, que en volumen e importancia temporalmente había sido el tercer exportador de lanas en el sur del Perú, ya en 1930 cayó víctima de la crisis económica mundial, y que Stafford y Ricketts, le siguieron a mediados de los años 30. Por supuesto que los autores tienen razón al afirmar que los comerciantes minoristas y los pequeños compradores de lana, los turcos y los rescatistas. fueron las víctimas más frecuentes de la difícil situación de mercado (p). 46) y que los mayoristas disponían de estrategias comerciales y posibilidades para disminuir los peligros de la crisis económica y traspasarlos hacia otros grupos sociales (p. 121). (El hecho re alzado por Burga y Reátegui, que Ricketts obtuviera mayores márgenes de ganancias en la época difícil de los años 20 que durante el auge de las exportaciones durante e inmediatamente después de la primera Guerra Mundial (p. 122), por cierto sólo debe entenderse como resultado de la estrategia comercial especial de Ricketts, que exportaba considerablemente menos lana, pero de calidad superior en esta época. Para las casas comerciales como Gibson y Stafford, que luchaban por mantener y ampliar su participación en el mercado, indudablemente era mucho más difícil seguir comprando sólo lana de óptima calidad y hacer bajar los precios de compra, mucho más de lo que habian bajado los precios de exportación). Pero lo más importante y decisivo era, que la mayoría de los mayoristas en Arequipa tenían fondos capitales relativamente débiles, 
al compararlos p.e. con casas comerciales importantes limeñas de esa época (Flores Galindo. Plaza, Oré, p. 59). Esto significaba que, al perdurar el estancamiento comercial, al perderse créditos otorgados a comerciantes y productores de lana o al hacerse especulaciones mercantiles fallidas, las reservas de capital de esta firmas rápidamente se agotaban y los mayoristas se acercaban al borde de la bancarrota.

Pero si el rendimiento de los negocios de mayoristas eran mucho más inseguros de lo que Burga y Reátegui suponen, entonces también es probable que sus inversiones en otros sectores económicos hayan tenido mayor importancia que la admitida por los autores. Respecto a la rentabilidad de las haciendas ganaderas, que evidentemente fueron un objetivo de inversión para los mayoristas, nuevamente se observa el estilo argumentativo contradictorio del libro que aqui se analiza. Por un lado, Burga y Reátegui, refiriéndose a Witold Kula, calculan la "renta feudal" de las haciendas andinas como una proporción entre el precio de venta de los productos de la hacienda y los costos de producción en dinero cuando pretenden demostrar la rentabilidad de las haciendas para los hacendados (p. 120). En cambio, cuando pretenden demostrar que las haciendas ganaderas, en comparación con el comercio mayorista, producian pocas ganancias, incluyen el valor de la tierra y del factor trabajo en sus cálculos. Solo así pueden argumentar que las ganancias en promedio del 6o/o, calculadas de esta manera para las haciendas, eran más bajas que las ganancias del 10o/o en la venta de productos manufacturados y las expectativas gananciales supuestamente aun más altas en la exportación de lanas, y que para Ricketts y los demás mayoristas, por lo tanto, habría sido irracional invertir en las haciendas (p. 125). Es decir, si se calculara la rentabilidad de las haciendas del sur peruano según el primer método, que pone énfasis en el modo operacional tradicional basado en la minimización de los costos monetarios de producción, entonces no parece tan seguro, que el comercio fuera necesariamente más lucrativo a largo plazo.

Contrariamente a lo que afirman Burga y Reátegui, los mayoristas, desde comienzos de los años 20, hicieron considerables inversiones de capitales en las haciendas ganaderas (Flores Galindo, Plaza, Oré, p. 60 y 67). Las familias Gibson, Rey de Castro, Muñoz Najar, Irigoyen von der Heyde y Ratti compraron grandes haciendas, especialmente en el departamento de Puno. La Sociedad Ganadera del Sur. S.A., fundada por Gibson en 1926, tenía un capital inicial de 137,500 libras peruanas y posteriormente fue propietaria de once haciendas en Puno con aproximadamente 100,000 hectáreas, la propiedad agrícola más grande en la sierra sudperuana (Expediente de Afectación, Sociedad Ganadera del Sur). 
El conflicto entre las casas comerciales en Arequipa y los hacendados tradicionales de la sierra, desde comienzos de los años 20 , no estaba determinado entonces primariamente, como piensan los autores (p. 108 y 184), por el problema del acceso de los colonos al mercado éste de todos modos, era relativamente libre, como hemos visto anteriormente. Los hacendados, más que nada, sentían su dependencia financiera de las casas comerciales como cada vez más opresora y pesada y veían el peligro, que esto pudiera conducir a la pérdida de sus tierras. Incluso los hacendados no afectados en forma inmediata por el peligro, temian las consecuencias de la penetración, en su esfera de producción, de intereses de capital que les parecian poderosísimos, fuera a través de los planes ostentosos de un coronel Stordy y la Peruvian Corporation o en la forma de compras de haciendas comparativamente modestas de los exportadores de Arequipa. Los hacendados tradicionales veían este aspecto - no en el acceso de sus colonos al mercado- el peligro de perder su control socio-político sobre la sociedad provincial, ya que creían que los complejos agrarios planeados o erigidos por empresas comerciales o de transportes cambiarian totalmente las relaciones de producción mismas y con ello, la base de su posición de poder (Belon, p. 13).

Estos temores, sin embargo, resultaron ser completamente exagerados. Los mayoristas, en sus haciendas, quisieron o pudieron crear complejos agrícolas modernos, intensivos de capitales, basados en el trabajo asalariado en escala mucho menor y un ritmo más lento de lo que la élite provincial tradicional, inmersa en el nacionalismo naciente de los años 20 y 30 , había temido.

Cabe anotar entonces, que las casas comerciales por cierto se esforzaban en diversificar sus inversiones, y no sólo tendiendo hacia la ganadería, sino también, aunque en menor escala, hacia otros sectores como la minería, el transporte, organismos bancarios y de seguros e incluso en la industria (Flores Galindo, Plaza, Oré, pp. 62-67). Es justamente esta diversificación de los intereses económicos la que caracteriza a las familias de la oligarquía regional en el sur del Perú desde fines de la Guerra del Pacífico. Por un lado, este tipo de inversiones fuera del ámbito comercial servía a los mayoristas para asegurar su posición económica de esta manera era posible compensar pérdidas en su sector. Pero por otro lado también correspondían a las necesidades sociales y políticas de una élite regional, cuya meta era afianzar la posición de poder que recién había logrado. Un pequeño grupo de exportadores de lana seguramente no habría sido capaz de desarrollarse como oligarquía regional bien definida, con ciertas pretensiones de exclusividad (vale decir, aislamiento frente a otros grupos sociales), si no hubiera tenido conexiones con los terratenientes, empresarios mineros y de transportes y con 
algunos sectores de la industria ligera elaboradora que recién nacía en el sur del país. Burga y Reátegui no han reconocido la importancia de este tipo de nexos, que no sólo se establecieron mediante inversiones en otros sectores sino también, y especialmente, a través de alianzas familiares y de clientela, factores que casi no aparecen en su libro. Enlaces familiares, como p.e. entre los Lira y los Harmsen, los López de Romaña y los Ugarteche y los Castresana, los Gibson y los Iriberry y los Rey de Castro unificaban intereses comerciales y terratenientes, pero a veces también conducían a alianzas entre comerciantes. Sería interesante investigar en qué medida se efectuaron alianzas entre familias de mayoristas y de hacendados ganaderos en Puno y las provincias vecinas de Cusco y Arequipa. Yo tengo la impresión que sólo unas pocas familias terratenientes de las haciendas ganaderas más grandes lograron establecer enlaces familiares con la oligarquía de Arequipa, la mayoría de los hacendados tenían relaciones de clientela con los comerciantes arequipeños, les vendía su lana, les compraba las mercancías importadas y obtenía créditos de ellos mediante una iuenta corriente.

Considerando estos aspectos, hay dos conceptos centrales de los autores, que pasan a tener una validez sólo restringida:

1. La opinión, que en el primer tercio de este siglo, los conflictos económicos y políticos en el sur del Perú se hayan manifestado principalmente como intereses de clase contrarios. Los autores en verdad describen, cómo se produjeron diversas alianzas de clase, $\mathrm{p}$. e. el interés común de exportadores y hacendados frente a la Peruvian Corporation, propietaria de los Ferrocarriles del Sur, y frente a los planes del Coronel Stordy y la misteriosa Peruvian Alpaca Company de monopolizar el comercio de esta lana e incluso organizar enormes latifundios con crianza de ovejas y alpacas al estilo de Argentina (pp. 57-59), pero por otra parte también relatan los conflictos entre los comerciantes exportadores y los hacendados, especialmente en años de crisis, en lo referente a los precios de la lana o a la actitud frente a movimientos de rebelión indígena. También narran que en diversas campañas, tanto los hacendados como los mayoristas siempre presentaban demagógicamente sus propios intereses como los de casi todas las capas sociales.

Nii opinión, sin embargo es, que los conflictos entre clanes familiares y lis redes clientelas integradas verticalmente, en general se superponían a lus conflictos económicos y sociales entre clases. Las diferencias políticas en el sur del Perú siempre se caracterizaban por rivalidades económicas y divergencias inmediatas de intereses entre miembros de la misma clase; así p.e... los conflictos entre los dueños de haciendas azucareras, Lira y López de Rumaña, entre las familias de hacendados Lizares Quiñones y Paredes en Azángaro (desde los años 50 hasta 80 del siglo pasado), o también los conflictus 
entre las casas comerciales Gibson y Stafford, que los mismos Burga y Reategui consideran como comercialmente incomprensibles (p. 146). Incluso al examinar asociaciones que se decían representantes de los intereses de ciertas clases sociales, como p.e. la Cámara de Comercio de Arequipa o la Sociedad Ganadera del departamento de Puno en 1921, me parece oportuno preguntarse escépticamente, si éstas no representaban también los intereses de alianzas familiares particulares y de sistemas de clientelas en contra de otros clanes familiares que socialmente no se diferenciaban de este grupo.

Todo esto no significa que no sea posible demostrar que existían intereses específicos de clase en la estructura social del sur del Perú en el primer tercio de este siglo, y que, por supuesto, los intereses relacionados con el comercio de exportación dominaban. Pero me parece calificativo y sintomático para una sociedad que se caracteriza por un grado insignificante de acumulación de capitales, por una clase socio-política dominante de indole oligárquica, por una fuerte dependencia vertical que trasciende las clases sociales y por el acceso al poder político limitado a una capa muy reducida de la sociedad que los conflictos de intereses precisamente no se articulan mayormente entre clases sociales políticamente organizadas y conscientes.

2. De esto también se desprende, que no es posible hacer una diferenciación tan nítida entre dos niveles comerciales de carácter casi contrario, un nivel capitalista y otro de presiones precapitalistas equivalente a la "negación del mercado", como lo sugieren Burga y Reátegui (pp. 72-73, 102103).

Por supuesto que se puede estar de acuerdo con los autores, cuando éstos describen cómo se realizaba la compra de lana de los campesinos a través de los rescatistas, en un ambiente de violencia, estafa y dependencia reforzada por relaciones de compadrazgo. Mas en este ámbito también existía competencia, como sabemos por las aciividades de lus diversos alcanzadores, empleados de comerciantes rivales de lanas yue acechaban a los campesinos que venían a la ciudad para vender su lani.

¿Y qué diferencia tan fundamental podía haber existido con el otro nivel de mercado, en el cual compraban y vendían hacendados, comerciantes locales de lanas y los mayoristas? ¿No sabemos, que los comerciantes tambićn trataban de estafar a los hacendados, manipulando las balanzas y pesas? ¿no era también una parte de la estrategia de los mayoristas, la de hacer depender a los hacendados de ellos, p.e. mediante créditos o -y esto lo creo probable, pero por el momento sólo puedo demostrarlo para el tiempo anterior a la Guerra del Pacífico- incluso mediante relaciones de compadrazgo? Appleby describe (pp. 70-71) el empeño de los exportadores de lana, de establecer relaciones comerciales a largo plazo con los hacendados, y que 
éstos, si eran "fieles" a una firma arequipeña, podian contar con concesiones o ventajas especiales p.e., precios algo más altos por sus lanas, que los precios habituales de mercado. Los mismos Burga y Reátegui citan (p.85). cómo el exportador Stafford, en 1926, trató de convencer a los hacendados de la región de Sicuani "con una buena champañada", para que le vendieran sus lanas. Los rescatistas usaban el aguardiente de caña para convencer a los campesinos, lo que evidentemente era más barato. Naturalmente que es cierto, que los hacendados por lo gęneral obtenían mejores precios por su lana que los campesinos. Pero esto no me parece indicio suficiente para hablar de esferas de mercado en principio diferentes; yo lo considero más bien una característica estructural de sociedades organizadas verticalmente por clientelas, vale decir, que la pertenencia a una capa social, tambićn inlluencia al mismo tiempo la posición de esa persona en el mercado frente a micmbros de otras capas (esto significa, que en sociedades marcadamente organizadas a través de clientelas y redes de familia no pueden existir contratos libremente concertados entre personas pertenecientes a diferentes capas sociales, un concepto básico del pensamiento económico liberalcapitalista). Es decir, que la "articulación de capitalismo y precapitalismo", que Burga y Reátegui pretenden diferenciar, no puede identificarse con diversas esferas de mercado. En el sur del Perú existía más bien un mercado de lanas estructurado jerárquicamente, en el cual la posición social de compradores y vendedores estaba altamente correlacionada con el nivel de comercialización, en el que alguien vendia lanas y el que también determinaba el nivel de los precios. Esto significa, que como los grandes hacendados firmaban directamente sus contratos con los mayoristas o sus agentes en los centros de mercado más grandes de la región productora de lanas, mientras que muchos campesinos vendian su lana a los rescatistas ambulantes o pequeños comerciantes, los hacendados, ya por esta razón ganaban más en sus ventas de lana.

Pero los aspectos capitalistas y precapitalistas en el comercio sudperuano se manifestaban en todos los niveles de mercado entre campesinos y mayoristas, a través de estrategias y mecanismos comerciales no equivalentes. Por ello repito: No me convence que se diga que "la relación del campesino con el rescatista misti era el encuentro de dos mundos diferentes, cada uno con su propia racionalidad y cosmovisión" (p. 104). El hecho que los campesinos orientaran sus ventas de lana en los precios de mercado, en el que los autores insisten en otra parte de su libro (p. 106), indudablemente indica, que éstos comprendian ciertos aspectos de la economia de mercado capitalista. 
Para finalizar, deseo hacer algunas observaciones referentes al método del trabajo de Burga y Reátegui. Los autores, sin problematizarlo, han partido de una casa comercial, del desarrollo de su volumen de ventas y sus ganancias, de la estructuración especial de sus relaciones comerciales, etc. para generalizar y deducir el ritmo y la estructura del comercio en todo el sur peruano. Considerando el valor heurístico único de la fuente para el tema tratado, este intento, en principio, me parece legítimo. Pero habría sido necesario evaluar claramente, si el destino de una firma comercial era representativo para todo el comercio sudperuano. Burga y Reátegui hacen esto solamente, casi casualmente, al referirse a ciertas estrategias comerciales de Ricketts referidas en cartas comerciales de la firma misma, que permiten hacer comparaciones con otros exportadores como Gibson o Stafford (pp. 79 y 100). Pero ¿qué pasa con el volumen y los precios de los negocios de lanas de Ricketts, con la distribución regional de este comercio, con el alcance de los cambios en las estructuras agrarias de producción como reacción a la exportación de lanas? ¿Podemos aceptar realmente, que el ritmo y las estructuras para todo el sur del Perú coincidían en su mayor parte con las reveladas a través del análisis de la contabilidad y correspondencia de Ricketts, siendo que Ricketts abarcaba como máximo el 15o/o de las exportaciones de lanas en el sur peruano y generalmente menos del 10o/o (vea Bedoya)? Para una serie de problemas, como p.e. los precios y el volumen de lana exportada, habrían existido métodos relativamente sencillos para examinar si los datos de Ricketts correspondían también a los valores generales para el sur del Perú. Para otras incógnitas, como p.e. la distribución del comercio en las varias subregiones, habría sido mucho más difícil, pero no imposible, establecer este tipo de correlación cuantitativa (p.e., revisando los ingresos fiscales de la alcabala o, un indicador más burdo, de la contribución industrial). Pero incluso para aquellas preguntas, para las que no habría sido posible o no habría tenido sentido establecer correlaciones cuantitativas, habría sido sumamente útil, si los autores al menos hubieran hecho reflexiones hipotéticas respecto a la fuerza informativa de los datos de Ricketts para las estructuras globales. Muchas afirmaciones o relaciones habrían ganado en valor, al delimitar los datos específicos para la firma Ricketts y los datos generales representativos para la región. Al periodizar p.e. los ritmos coyunturales, cuyos cortes coinciden plenamente tanto para Ricketts como para el comercio en general (aquí resultó interesante el manejo por los autores del período 1922 hasta 1935 como un todo, sin corte al comenzar la crisis económica mundial), la fase 1895-1914 habría encontrado otra interpretación, si se hubiera interpretado en un contexto a largo plazo del comercio de lanas desde los años 1850, o incluso a partir de 1820. Los dos decenios pre- 
cedentes a la primera Guerra Mundial no fueron para todo el comercio de lanas del sur del Perú, como para Ricketts, un período de aumento relativamente rápido del volumen de exportación, con precios estables, que los autores consideran bajos. Al terminar la fase de recuperación posterior a la Guerra del Pacífico alrededor de 1890, el volumen de exportación siguió creciendo más bien a un ritmo muy lento hasta 1914. En cambio, al comparar los 19 años siguientes con los decenios críticos de 1873 hasta 1895 , se constata que hubo aumentos considerables en los precios, medidos en Soles moneda nacional, o más aún, en moneda boliviana.

También con respecto al mejoramiento de la calidad de las lanas mediante la crianza selectiva, que Burga y Reátegui entienden como una clara aceptación de las exigencias del mercado mundial por parte de los productores (p. 90), los autores podrían haber presentado mucho menos contradicciones, si hubieran diferenciado entre los objetivos de la estrategia comercial de Ricketts, que ya desde mediados de los años 20 había exigido a sus proveedores un 60o/o de lana blanca de alpaca y un 40o/o de lana merino, y la calidad media real de la lana ofrecida por haciendas y campesinos. Todavía en 1929, el porcentaje de lana merino exportada desde el sur peruano sólo equivalía a un 13,10/o (véase Estadistica del Comercio especial. . . 1929). Es probable que la cuota de lana alpaca haya sido algo mayor (las estadísticas de exportación aquí no hacen diferenciación entre calidades). Pero es evidente que el mejoramiento de la calidad de la lana -especialmente hasta 1920 y contrariamente a la opinión que los autores obtuvieron a través de la correspondencia de la firma Ricketts-se limitó sólo a contadas haciendas y a las crianzas de algunos campesinos. Es decir, que al recurrir a datos globales de comparación, en este caso casi se habría llegado a una inversión de la tesis lograda a través de la correspondencia de Ricketts: vale decir, el grado mínimo de adaptación de la mayoría de los productores, al menos hasta 1920, a las exigenciàs de calidad del mercado mundial.

Este no ha sido un análisis crítico de un libro en el sentido habitual de la palabra. Así, no me he detenido en analizar una serie de aportes valiosos de este libro, como p.e. la discusión sobre la competencia existente entre las casas comerciales extranjeras residentes en Lima y las fábricas textiles para conquistar el mercado en el sur del Perú, y el primer examen sistemático del rol del dinero boliviano, especialmente en Puno. Indudablemente, Burga y Reátegui han logrado con esta monografía, que comprendamos mejor una serie de mecanismos importantes y relaciones del ámbito económico dominado por Arequipa. Pero por cierto que habría sido correcto anotar, no solamente en la introducción, que muchos aspectos del sistema comercial que 
lus autores trataron, ya habían sido analizados por otros autores, especialmente por Gordon Appleby, pero también. por Benjamín Orlove.

Ln este análisis sólo se pretendía discutir algunos problemas conceptuales y metodológicos del libro, que considero de suma importancia para una interpretación convincente y trascendental del desarrollo social y económico en el sur del Perú. Aquí -sin considerar una serie de errores empíricos los que se podrian haber evitado -. el libro presenta flaquezas: los autores no lograron ofrecer una imagen convincente y sin contradicciones internas, de las relaciones entre el comercio y el sistema de producción, la relación entre oligarquía y pequeños comerciantes, entre hacendados y campesinos y final.mente, de la articulación del "capitalismo y precapitalismo".

\section{BIBLIOGRAFIA}

\section{APPLEBY, Gordon}

1978 "Exportation and its Aftermath: The Spatioeconomic Evolutio of the Regional Marketin System in Highland Puno, Peru". Ph.D. Diss., Stanford University.

BEDOY $\wedge$, Guillermo

1923....1938 Estadística de exportación de la región del Sur del Perí por la vía de ilullendo, Mollendo.

BI:LON Y BARRIONULVO, Carlos

$19+5$ La industria sanadera del departamento de Puno y su economía social, Arcyuipa, Tipografía $\lambda$ costa.

IFLORES CisliNDU, Allertu: Oré, Teresajplaza, Orlando

1978 "()ligaryufa y capital comercial en el Sur Peruano, 1870-1930", Ponencia prescontada al $V$ Simposio de historia económica de America Latina iCL.ACSU), Lima.

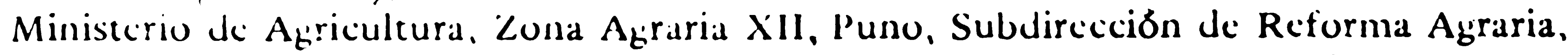
s.f. Lipecliente de Alectación, Suciedad Cianadera del Sur, manuscrito.

Mlinisterio de llacienda y Comercio, Superintendencia General de Aduanas

1930 Estudística del comercio c'special del Perú ell el año de 1929, Callao.

ORL()VL, BenjamIn

1977 Alpaca, Shcep and Men: The Wool Export Economy and Regional Socicty' in Southern Peru, New York. 